\title{
A Major Role for Neutrophils in Experimental Bullous Pemphigoid
}

\author{
Zhi Liu, ${ }^{\star}$ George J. Giudice, ${ }^{\star \ddagger}$ Xiaoye Zhou, ${ }^{\star}$ Susan J. Swartz, ${ }^{\star}$ James L. Troy, ${ }^{\star}$ Janet A. Fairley, ${ }^{\star \S}$ Gerd O. Till," \\ and Luis A. Diaz ${ }^{\star \S}$ \\ *Department of Dermatology and ${ }^{\ddagger}$ Department of Biochemistry, Medical College of Wisconsin, Milwaukee, Wisconsin 53226; ${ }^{\S}$ Veterans \\ Affairs Medical Center, Milwaukee, Wisconsin 53295; and ${ }^{\|}$Department of Pathology, University of Michigan Medical School, Ann Arbor, \\ Michigan 48109
}

\begin{abstract}
Bullous pemphigoid (BP) is an inflammatory subepidermal blistering disease associated with an IgG autoimmune response to the hemidesmosomal protein, BP180. Using a passive transfer mouse model, our group has shown previously that antibodies to the murine BP180 (mBP180) ectodomain are capable of triggering a blistering skin disease that closely mimics human BP. In this study, we investigated the role of neutrophils in the immunopathogenesis of this disease model. BALB/c mice depleted of circulating neutrophils by treatment with neutrophil-specific antibodies were no longer susceptible to the pathogenic effects of anti-mBP180 IgG. IgG and complement were deposited at the dermalepidermal junction of these animals, but there was no evidence of inflammatory infiltration or blistering. C5-deficient mice, which are resistant to the pathogenic activity of antimBP180 IgG, could be made susceptible to this IgG-mediated blistering disease by intradermal administration of a neutrophil chemoattractant, IL-8 or C5a. Intraperitoneal injection of IL-8, which sequesters neutrophils in the peritoneal cavity, interferes with anti-mBP180-induced neutrophilic infiltration of the skin and prevented the development of BP disease in $\mathrm{BALB} / \mathrm{c}$ mice. These findings provide the first direct evidence that neutrophils recruited to the skin via a C5-dependent pathway play an essential role in subepidermal blister formation in experimental BP, and suggest new directions for disease intervention. (J. Clin. Invest. 1997. 100:1256-1263.) Key words: autoimmunity • mouse model $\bullet$ hemidesmosome $\cdot$ inflammation $•$ basement membrane zone
\end{abstract}

\section{Introduction}

Bullous pemphigoid (BP $)^{1}$ is an acquired autoimmune disease of the skin characterized by subepidermal blisters and autoantibodies against two hemidesmosomal antigens, BP230

Address correspondence to Zhi Liu, Ph.D., Department of Dermatology, Medical College of Wisconsin, 8701 Watertown Plank Road, Milwaukee, WI 53226. Phone: 414-456-4087; FAX: 414-266-8673; E-mail: zhiliu@post.its.mcw.edu

Received for publication 3 February 1997 and accepted in revised form 30 May 1997.

1. Abbreviations used in this paper: $\mathrm{BMZ}$, basement membrane zone; BP, bullous pemphigoid; DEJ, dermal-epidermal junction; GST, glutathione $S$-transferase; H/E, hematoxylin and eosin; IF, immunofluorescence; mBP180, murine BP180 antigen; MPO, myeloperoxidase.

The Journal of Clinical Investigation

Volume 100, Number 5, September 1997, 1256-1263

http://www.jci.org
(BPAG1) and BP180 (BPAG2) (1). These antihemidesmosomal autoantibodies are found in the circulation of patients, and can be detected, along with complement proteins, bound to the dermal-epidermal junction (DEJ) of perilesional skin. The skin lesions of these patients show detachment of basal keratinocytes from the underlying dermis leading to subepidermal blistering. The separation of the DEJ occurs at the level of the lamina lucida $(1,2)$. Various inflammatory cells, including eosinophils $(3,4)$, neutrophils (5), lymphocytes (6), and monocyte/macrophages $(7,8)$, have been identified in the upper dermis of lesional areas in patients with BP. Accumulation and morphological changes of mast cells have also been found in these areas $(9,10)$. The blister cavity typically contains fibrin, eosinophils, and neutrophils (2). Similar skin lesions are observed in the pregnancy-associated nonviral disorder, herpes gestationis (11).

Recently, our laboratory has developed an experimental model of BP which involves the passive transfer of antimBP180 antibodies into neonatal BALB/c mice (12). The BP mouse model reproduces the key immunopathological features of this human autoimmune disease, i.e., IgG and complement deposition at the DEJ, inflammatory infiltration of the upper dermis, and subepidermal blistering. Several lines of evidence demonstrated that the pathogenicity of anti-mBP180 antibodies is dependent on complement activation (13). Mice depleted of complement (either genetically or by pretreatment with cobra venom factor) did not develop blisters after injection with pathogenic rabbit anti-mBP180 antibodies. In addition, it was shown that the $\mathrm{F}\left(\mathrm{ab}^{\prime}\right)_{2}$ fragments generated from the anti-mBP180 IgG exhibited no pathogenic activity in the mouse model. Manipulations that interfered with complement activation also resulted in a drastic reduction of neutrophil influx into the dermis of the animals (13), suggesting that neutrophils may play a key role in blister formation in the experimental $\mathrm{BP} / \mathrm{HG}$ model.

Previous findings reported by Gammon et al. (14) using an in vitro model of BP led to the hypothesis that subepidermal blistering in BP is dependent upon antibody binding to an antigen of the DEJ, complement activation and an inflammatory infiltration of lesional skin. These investigators showed that, when cryosections of normal human skin were incubated in an organ culture system with a BP serum plus an exogenous source of complement and human peripheral blood leukocytes, the inflammatory cells migrated and attached to the DEJ in these sections. Moreover, the sections developed focal dermalepidermal separation resembling early BP lesions (15), but only if the inflammatory cells were present. These observations provided the first indication that inflammatory cells, such as the neutrophil, may play a key role in the immunopathogenesis of $\mathrm{BP} / \mathrm{HG}$.

In this study, we investigated the possibility that the neutrophil is a key cellular element responsible for triggering dermal-epidermal separation in the murine BP model. Using various experimental approaches, we showed that blockage of 
neutrophil recruitment resulted in the neutralization of the pathogenic activity of anti-mBP180 antibodies in mice. Furthermore, we were able to circumvent the pathogenic block exhibited by C5-deficient mice by intradermal administration of a neutrophil chemoattractant. These results clearly demonstrate that neutrophil activation is an essential element in the pathogenesis of blister formation in experimental $\mathrm{BP} / \mathrm{HG}$.

\section{Methods}

Laboratory animals. Breeding pairs of BALB/c, B10-D2-NSN (C5sufficient strain), and B10-D2-OSN (C5-deficient strain) mice were purchased from Jackson Laboratories (Bar Harbor, ME) and maintained at the Medical College of Wisconsin Animal Resource Center. Neonatal mice (24-36 h old with body weights between 1.4 and $1.6 \mathrm{~g}$ ) were used for passive transfer experiments.

Preparation of pathogenic rabbit anti-murine $I g G$. The preparation of recombinant $\mathrm{mBP} 180$ and the immunization of rabbits were performed as previously described (12). Briefly, a segment of the mBP180 antigen encompassing amino acids $495-643$ of the ectodomain of this protein (using the numbering system of Li et al. [16]) was expressed as a glutathione $S$-transferase (GST) fusion protein using the pGEX prokaryotic expression system (Pharmacia LKB Biotechnology, Piscataway, NJ). This fusion protein, designated GST-mBP180ABC, was purified to homogeneity by affinity chromatography (17). New Zealand White rabbits were immunized with the purified $\mathrm{mBP} 180$ fusion protein, and the $\mathrm{IgG}$ fraction from the sera (referred to as R50, R621, and R622) was purified as previously described $(12,18)$. The $\operatorname{IgG}$ fractions were concentrated, sterilized by ultrafiltration, and the protein concentrations were determined by $\mathrm{OD}_{280}[\mathrm{E}(1 \%, 1 \mathrm{~cm})=13.6]$. The titers of rabbit anti-mBP180 antibodies in the rabbit sera and in the purified IgG fractions were assayed by indirect immunofluorescence (IF) using mouse skin cryosections as substrate. These antibody preparations were also tested by immunoblotting for reactivity with the GST-mBP180ABC fusion protein. The IF and immunoblotting techniques have been reported elsewhere (12). The pathogenicities of these $\mathrm{IgG}$ preparations were tested by passive transfer experiments, as described below. The immunologic characteristics of rabbit anti-mBP180 antisera R50, R621, and $\mathrm{R} 622$ are listed in Table I.

Induction of experimental BP and animal evaluation. A 50- $\mu$ l dose of sterile IgG in PBS was administered to neonatal mice by intradermal (2.5 mg IgG/g body wt) or intraperitoneal injection $(5.0 \mathrm{mg} \mathrm{IgG/g}$ body wt). The injection techniques have been described elsewhere $(12,19,20)$. The skin of neonatal mice from the test and control groups was examined $12 \mathrm{~h}$ after the IgG injection. The extent of cuta-

Table I. The Immunologic Profiles of Rabbit Anti-murine BP180 IgG Preparations

\begin{tabular}{lcccc}
\hline Rabbit IgG & $\begin{array}{c}\text { Immunoblotting } \\
\text { with mBP180* }\end{array}$ & $\begin{array}{c}\text { Mouse BMZ } \\
\text { staining (IIF) }\end{array}$ & $\begin{array}{c}\text { In vivo BMZ } \\
\text { deposition (DIF) }\end{array}$ & $\begin{array}{c}\text { Pathogenic } \\
\text { activity }\end{array}$ \\
\hline R50 & + & - & - & - \\
R622 & + & + & + & - \\
R621 & + & + & + & +
\end{tabular}

* Purified GST-mBP180ABC was run on a $12 \%$ SDS-PAGE gel, electrotransferred to a nitrocellulose filter membrane, and probed with rabbit anti-mBP180 IgG $(2 \mu \mathrm{g} / \mathrm{ml})$. ${ }^{\ddagger}$ BALB/c mouse skin cryosections were labeled with rabbit anti-mBP180 IgG $(2 \mu \mathrm{g} / \mathrm{ml})$ and visualized with FITC-conjugated goat anti-rabbit IgG. ${ }^{\S}$ Direct IF of skin cryosections of BALB/c mice injected with rabbit anti-mBP180 IgG using FITC-conjugated goat anti-rabbit IgG. "Pathogenic activity of the rabbit anti$\mathrm{mBP} 180 \mathrm{IgG}$ was determined by passive transfer experiments using neonatal BALB/c mice (see reference 18). neous disease was scored as follows: (-), no detectable skin disease; $1+$, mild erythematous reaction with no evidence of the "epidermal detachment" sign (this sign was elicited by gentle friction of the mouse skin which, when positive, produced fine, persistent wrinkling of the epidermis); $2+$, intense erythema and epidermal detachment sign involving $10-50 \%$ of the epidermis in localized areas; and $3+$, intense erythema with frank epidermal detachment sign involving $>50 \%$ of the epidermis. The animals were then killed and the following specimens were obtained. Skin sections were taken for light microscopy (hematoxylin and eosin [H/E] staining) and for direct IF analysis to detect rabbit $\mathrm{IgG}$ and mouse $\mathrm{C} 3$ deposition at the basement membrane zone (BMZ). Sera of injected animals were obtained for indirect IF assay to determine the circulating titers of anti-mBP180 IgG. Direct and indirect IF analyses were performed as previously described $(12,19,20)$. Monospecific FITC-conjugated goat antirabbit IgG was obtained commercially (Kirkegaard \& Perry Laboratories Inc., Gaithersburg, MD). Monospecific goat anti-mouse C3 was purchased from Cappel Laboratories (Durham, NC).

$P M N$ depletion and injection procedures. A polyclonal rabbit antimurine PMN antibody, AI-A31140, which selectively depletes mouse neutrophils in vivo, was purchased from Accurate Chemical \& Scientific Corp. (Westbury, NY). Mice were depleted of PMN by two intraperitoneal injections of AI-A31140 (10 $\mu \mathrm{g} \mathrm{IgG} \mathrm{in} 50 \mu \mathrm{l}$ PBS per injection) given $12 \mathrm{~h}$ apart. The control groups received either $2 \times 50 \mu \mathrm{PBS}$ or normal rabbit IgG $(10 \mu \mathrm{g} \mathrm{IgG} \mathrm{in} 50 \mu \mathrm{l}$ PBS $)$. Neutrophil levels in circulation were monitored by direct cell counting of blood smears stained with Wright dye (Baxter Diagnostics Inc., McGaw Park, IL) and by myeloperoxidase (MPO, a neutrophil cell marker) assay (21, 22). Both experimental and control groups of animals received one 50 $\mu l$, i.d. $(2.5 \mathrm{mg} / \mathrm{g}$ body wt/d) or i.p. ( $5 \mathrm{mg} / \mathrm{g}$ body wt/d) injection of rabbit anti-mBP180 IgG, $12 \mathrm{~h}$ after anti-PMN treatment. The animals were then killed, and the skin and sera of mice from the test and control groups were examined $12 \mathrm{~h}$ later as described above.

IL-8 and C5 a pretreatment of C5-deficient mice. Recombinant human IL-8 (R\&D Systems, Minneapolis, MN) and recombinant human C5a (Sigma Chemical Co., St. Louis, MO) were stored at 500 $\mu \mathrm{g} / \mathrm{ml}$ in sterile PBS. IL-8 was injected either intraperitoneally or intradermally. A single intraperitoneal injection of IL-8 (50 ng in $50 \mu \mathrm{l}$ PBS) or an equivalent amount of BSA was given into neonatal BALB/c mice 60 min before intradermal injection of rabbit anti-mBP180 IgG $(2.5 \mathrm{mg} / \mathrm{g}$ body wt in $50 \mu \mathrm{l})$. For intradermal applications, IL-8 or C5a (50 ng in $50 \mu \mathrm{l}$ PBS) or an equivalent mass amount of BSA was injected into C5-deficient mice along with anti-mBP180 $\operatorname{IgG}(2.5 \mathrm{mg} / \mathrm{g}$ body wt in $50 \mu \mathrm{l}$ ). Control animals received an equivalent amount of normal rabbit IgG, in place of the anti-mBP180 IgG.

Quantitation of skin site PMN accumulation. Tissue MPO activity in skin sites of the injected animals was assayed as described $(21,22)$. A standard reference curve was first established using known concentrations of purified MPO. The skin samples were extracted by homogenization in an extraction buffer containing $0.1 \mathrm{M}$ Tris-Cl, $\mathrm{pH}$ 7.6, $0.15 \mathrm{M} \mathrm{NaCl}, 0.5 \%$ hexadecyl trimethylammoniumbromide. MPO activity in the supernatant fraction was measured by the change in optical density at $460 \mathrm{~nm}$ resulting from decomposition of $\mathrm{H}_{2} \mathrm{O}_{2}$ in the presence of $o$-dianisidine. MPO content was expressed as units of MPO activity per milligram of protein. Protein concentrations were determined by a dye binding assay (Bio-Rad Laboratories, Richmond, CA) using BSA as a standard.

Statistical analysis. The data were expressed as mean \pm SEM and were analyzed using the Student's paired $t$ test. $P<0.05$ was considered significant.

\section{Results}

Time course of pathogenic IgG deposition, complement activation, neutrophilic infiltration, and dermal-epidermal separation. The chronology of events leading to dermal-epidermal separation after intradermal or intraperitoneal injection of 
Table II. Time Course of BMZ Deposition of Pathogenic IgG, Complement Activation, Circulating IgG Titer, and Dermal-Epidermal Separation

\begin{tabular}{lcccccc}
\hline $\begin{array}{l}\text { Hours after } \\
\text { intradermal } \\
\text { injection }\end{array}$ & $\begin{array}{c}\text { IgG deposition } \\
\text { (DIF) }\end{array}$ & $\begin{array}{c}\text { C3 deposition } \\
\text { (DIF) }\end{array}$ & IgG titer (IIF) & Clinical & H/E \\
\hline 0 & - & - & 0 & - & - \\
1 & \pm & \pm & $1: 80$ & - & - \\
2 & + & + & $1: 160$ & - & - \\
4 & + & + & $1: 640$ & - & + \\
8 & + & + & $1: 5120$ & - & + \\
12 & + & + & $1: 5120$ & + & + \\
\hline
\end{tabular}

Neonatal BALB/c mice were killed at different time points after intradermal injection of pathogenic anti-BP180 IgG. Skin sections were taken for light microscopy $(\mathrm{H} / \mathrm{E})$ and direct IF $(D I F)$ analysis to detect BMZ-bound rabbit IgG and murine $\mathrm{C} 3$. Circulating rabbit IgG levels were determined by indirect IF $(I I F)$ using mouse skin cryosections as substrate. In the last column, + means blister formation detected by clinical examination or $\mathrm{H} / \mathrm{E}$ staining; - means no detectable dermal-epidermal separation.

pathogenic anti-mBP180 IgG (R621) was documented over a $12-\mathrm{h}$ period. Five animals were used at each time point. The mode of administration of anti-mBP180 IgG had little effect on the time course of subsequent events, except for the fact that the presentation of findings after intraperitoneal injection of antibody was delayed by $\sim 2 \mathrm{~h}$ relative to findings associated with an intradermal injection of this $\mathrm{IgG}$.

After intradermal injections, anti-mBP180 IgG appeared in the circulation within $1 \mathrm{~h}($ titer $=1: 80)$ and reached a peak at $8 \mathrm{~h}$ (titer $=1: 5,120$ ). Deposition of rabbit $\mathrm{IgG}$ and murine $\mathrm{C} 3$ at the cutaneous BMZ was first observed at $2 \mathrm{~h}$ as determined by direct IF (Table II). Histological examination of the skin of mice revealed an early stage of dermal-epidermal separation at the 4-h time point, while relatively broad subepidermal vesicles could be seen at $8 \mathrm{~h}$ and complete detachment of the epidermis from the dermis occurred at $12 \mathrm{~h}$ after intradermal IgG injections (Fig. $1 \mathrm{~A}$ ). The first evidence of neutrophil recruitment to the skin site, as determined by MPO activity, was obtained between 2 and $3 \mathrm{~h}$ after intradermal injection, followed by a progressive increase over the next $5 \mathrm{~h}$, reaching a peak at 8 h (Fig. $1 B$ ).

Intraperitoneal injection of neutrophil-depleting antibodies resulted in neutropenia and resistance of neonatal $B A L B / c$ mice to pathogenic anti-mBP180 antibodies. In this experiment, we first established the degree of neutropenia in peripheral blood of neonatal BALB/c mice injected with rabbit anti-murine PMN antibodies. Two intraperitoneal injections of PMN-specific IgG $(10 \mu \mathrm{g}$ IgG in $50 \mu \mathrm{l}$ PBS per injection) into neonatal mice $(n=9)$ resulted in a profound neutropenia, with $<200$ $\mathrm{PMN} / \mathrm{mm}^{3}$ peripheral blood as determined by cell counting of

A
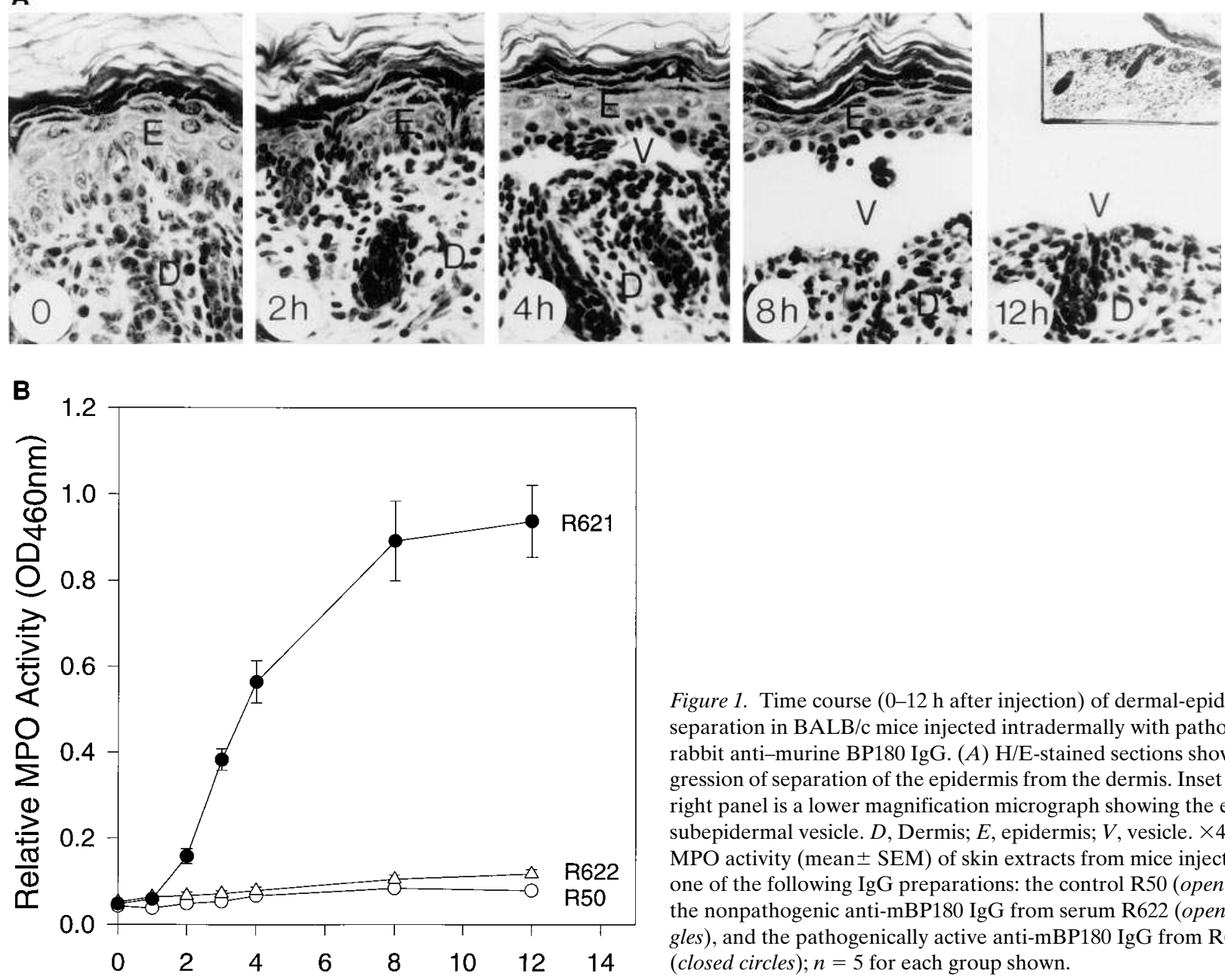

Figure 1. Time course (0-12 h after injection) of dermal-epidermal separation in $\mathrm{BALB} / \mathrm{c}$ mice injected intradermally with pathogenic rabbit anti-murine BP180 IgG. (A) H/E-stained sections showing progression of separation of the epidermis from the dermis. Inset in the far right panel is a lower magnification micrograph showing the edge of a subepidermal vesicle. $D$, Dermis; $E$, epidermis; $V$, vesicle. $\times 400$. (B) MPO activity (mean \pm SEM) of skin extracts from mice injected with one of the following IgG preparations: the control R50 (open circles); the nonpathogenic anti-mBP180 IgG from serum R622 (open triangles), and the pathogenically active anti-mBP180 IgG from R621 (closed circles); $n=5$ for each group shown. 


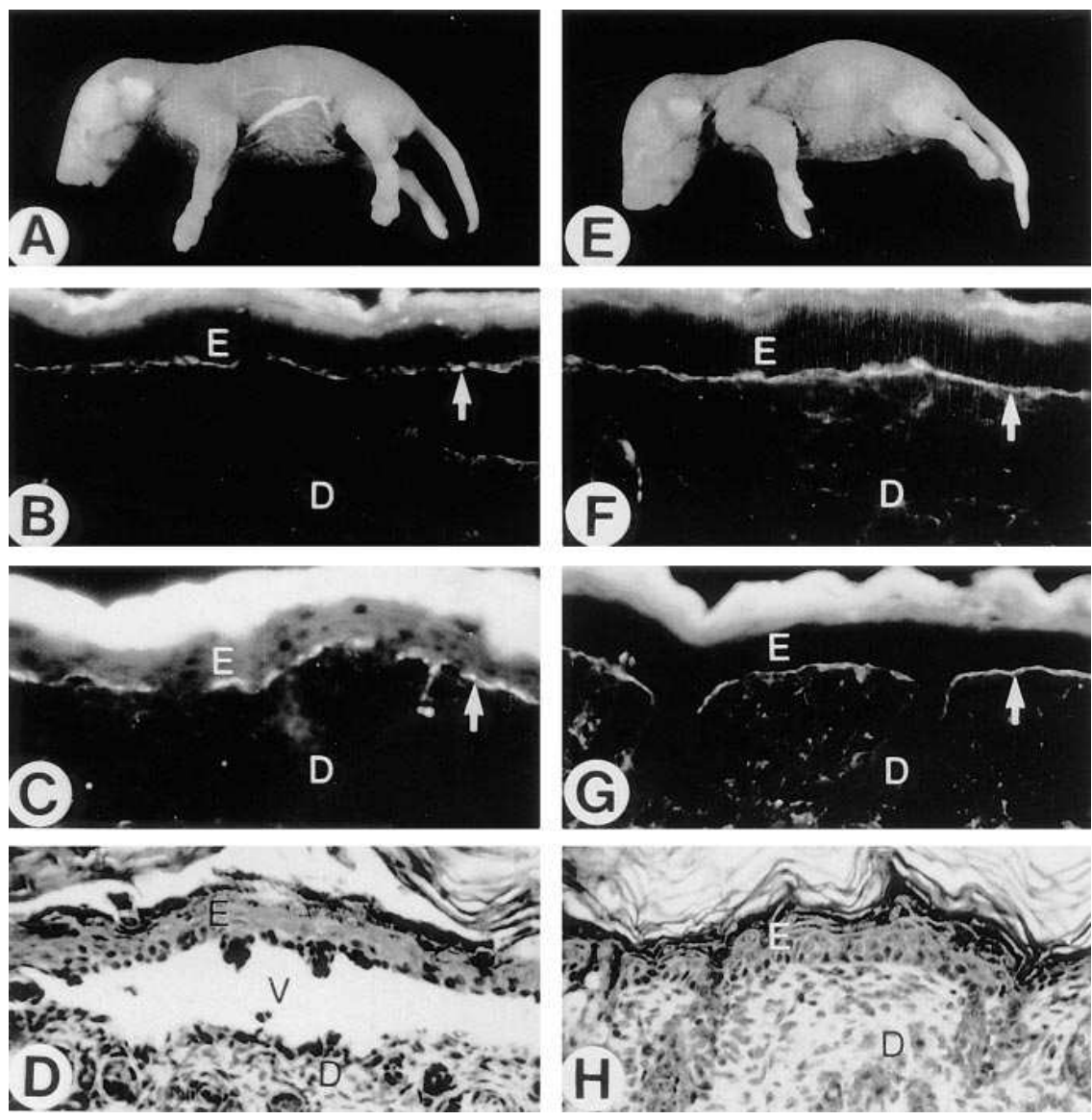

Figure 2. Clinical, IF, and histological examination of neonatal BALB/c mice pretreated with neutrophil-depleting antibodies and then injected with pathogenic rabbit anti-mBP180 IgG. The anti-mBP180 IgG induced extensive blistering disease $(A)$. The skin of these animals showed linear deposition of rabbit IgG $(B)$ and murine $\mathrm{C} 3(C)$ at the $\mathrm{BMZ}$, as determined by direct IF. H/E staining revealed epidermal-dermal separation with a neutrophilic infiltrate $(D)$. In contrast, neonatal BALB/c mice depleted of neutrophils and injected intradermally with pathogenic rabbit antimurine BP180 IgG showed no evidence of skin disease $(E)$. Direct IF demonstrated BMZ deposition of rabbit $\operatorname{IgG}(F)$ and murine C3 $(G)$. Histologic examination showed no epidermal-dermal separation and little inflammation $(H) . D$, Dermis; $E$, epidermis; $V$, vesicle; arrow, site of antibody labeling. $\times 400$. blood smears and MPO assay results (data not shown). This level persisted up to $24 \mathrm{~h}$. In contrast, control animals injected with the same volume of PBS or control IgG exhibited normal levels of circulating PMN.

Mice pretreated with either anti-PMN $(n=9)$ or control IgG $(n=9)$ were then injected with the pathogenic antimBP180 IgG (R621). The results for these experiments are shown in Figs. 2 and 3 (also see Table III). As expected, control animals (those injected with R621 IgG after pretreatment with either PBS or normal rabbit IgG in place of the anti-PMN antibody) developed extensive blistering (Fig. $2 A$ ). The skin of these injected animals was markedly erythematous and, upon gentle friction, developed persistent epidermal wrinkling due to epidermal detachment. Histologic examination revealed dermal-epidermal separation and neutrophilic infiltration of the dermis (Fig. $2 \mathrm{D}$ ), and direct IF showed deposition of rabbit IgG and murine $\mathrm{C} 3$ at the epidermal side of the vesicle (Fig. $2, B$ and $C$ ). In contrast, mice depleted of circulating neutrophils by pretreatment with anti-PMN antibodies showed no signs of blister formation after injection of pathogenic rabbit anti-mBP180 IgG (R621), despite the fact that these animals had high levels of circulating anti-mBP180 antibodies and showed deposition of IgG and $\mathrm{C} 3$ at the DEJ (Fig. 2, E-G). Histologic examination revealed no subepidermal separation nor neutrophilic infiltration of the dermis (Fig. $2 H$ ).

These findings were substantiated by the more quantitative assessment of neutrophilic infiltration which involved determination of tissue MPO activity levels (Fig. 3). The skin of posi- tive control mice (pretreated with either PBS or normal rabbit $\mathrm{IgG}$, in place of the anti-PMN antibody, and then injected with pathogenic R621 IgG) showed elevated levels of tissue extractable MPO activity $(0.888 \pm 0.036 \mathrm{U} / \mathrm{mg}$ protein $)$, which indicated the presence of neutrophils. In contrast, PMN-depleted animals injected with the pathogenic anti-mBP180 IgG showed significantly lower levels of MPO activity in their skin $(0.299 \pm 0.028 \mathrm{U} / \mathrm{mg}$ protein, $P<0.001)$. These data, together with those from histological examination, demonstrated that blocking PMN recruitment into the DEJ site is correlated with a neutralization of the pathogenic activity of the rabbit antimBP180 antibodies, thus suggesting that PMNs may play an essential role in blister formation in experimental BP.

Intraperitoneal injection of IL-8 reduces tissue PMN accumulation and inhibits blister formation in normal $B A L B / c$ mice treated with pathogenic anti-mBP180 IgG. Intravascular injections of IL- 8 have been used to sequester PMNs into the venules of animals, thus inhibiting neutrophil accumulation at sites of acute inflammation $(23,24)$. We applied a modification of this strategy (sequestration of neutrophils in the peritoneal cavity of mice) to further investigate the role of neutrophils in experimental BP. A group of eight neonatal BALB/c mice received a single intraperitoneal injection of IL-8 (10 ng/g body wt in $100 \mu \mathrm{l}$ of PBS), while a control group of age- and weightmatched mice received $10 \mathrm{ng} \mathrm{BSA} / \mathrm{g}$ body wt in $100 \mu \mathrm{l}$ of PBS. After $60 \mathrm{~min}$, each animal received an intradermal injection of anti-mBP180 IgG ( $2.5 \mathrm{mg} / \mathrm{g}$ body wt). Control mice, without IL-8 pretreatment, developed extensive blisters $12 \mathrm{~h}$ after the 


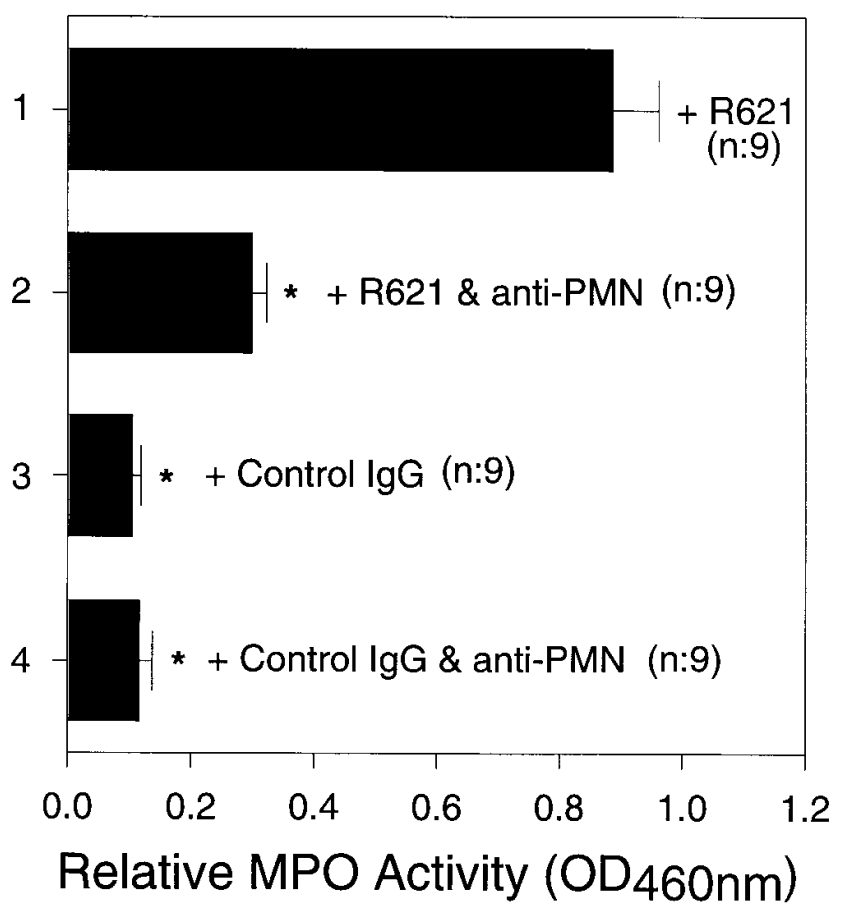

Figure 3. Systemic treatment with an anti-PMN antibody depletes circulating neutrophils and abolishes dermal-epidermal blistering in $\mathrm{BALB} / \mathrm{c}$ mice. The negative control animals (bars 3 and 4 ) were injected intradermally with normal rabbit $\operatorname{IgG}(2.5 \mathrm{mg} / \mathrm{g}$ body wt $)$ while the positive and anti-PMN-treated groups (bars 1 and 2) received an intradermal injection of pathogenic anti-BP180 IgG, R621 (2.5 $\mathrm{mg} / \mathrm{g}$ body wt). Tissue MPO activity (mean \pm SEM) in skin at the injection site was determined $12 \mathrm{~h}$ after IgG administration. $n=9$ for each group. ${ }^{*} P<0.001$, Student $t$ test for paired samples (bars 2, 3, or 4 vs. 1 ).

anti-mBP180 IgG injection (Table III). These blisters were subepidermal, as determined by histological examination, and were accompanied with neutrophilic infiltration of the dermis. In contrast, neonatal mice that received an intraperitoneal injection of IL- 8 resulted in significant decrease of neutrophils in

Table III. Summary: The Role of Neutrophils in BP Blister Formation

\begin{tabular}{lcccc}
\hline \multicolumn{1}{c}{ Host mice } & IgG injected & $\begin{array}{c}\text { Chemoattractant } \\
\text { injected }\end{array}$ & $\begin{array}{c}\text { Number of } \\
\text { mice }\end{array}$ & $\begin{array}{c}\text { Disease } \\
\text { activity* }\end{array}$ \\
\hline BALB/c & R621 & - & 28 & $3+$ \\
BALB/c & R621 + A31140 & - & 9 & - \\
C5-sufficient & R621 & - & 9 & $3+$ \\
C5-deficient & R621 & - & 17 & - \\
& R621 & i.d. IL-8 & 12 & $3+$ \\
& R621 & i.d. C5a & 8 & $3+$ \\
& R622 & i.d. IL-8 & 8 & $3+$ \\
& R50 & i.d. IL-8 & 8 & - \\
& R621 & i.p. IL-8 & 8 & -
\end{tabular}

*Disease activity is scored on a scale of - to $3+.-$ means no detectable skin lesion; $3+$ means intense erythema with frank epidermal detachment sign involving $>50 \%$ of the epidermis in the IgG-injected site. See Methods for details.

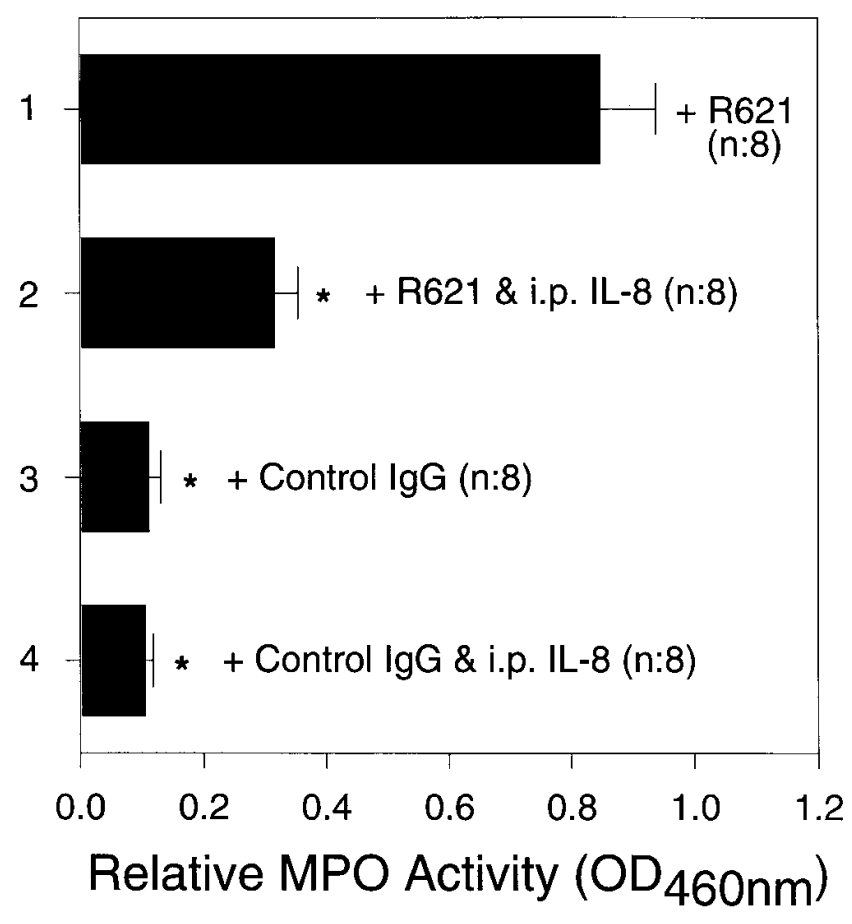

Figure 4. Intraperitoneal injection of IL-8 blocks dermal-epidermal separation by inhibiting neutrophilic infiltration. Neonatal BALB/c mice receiving an intraperitoneal injection of either BSA (bars 1 and 3 ) or IL-8 (bars 2 and 4) were given an intradermal injection of either pathogenic anti-BP180 IgG, R621 (bars 1 and 2), or normal control IgG (bars 3 and 4 ). Tissue MPO activity (mean \pm SEM) in skin at the injection site was determined $12 \mathrm{~h}$ after IgG administration. $n=8$ for each group. $* P<0.001$, Student $t$ test for paired samples (bars 2,3 , or 4 vs. 1$)$.

peripheral blood and became resistant to the pathogenic effects of anti-mBP180 IgG (Table III). The skin of these animals showed no evidence of subepidermal vesiculation and an absence of neutrophilic infiltration. Quantitation of intradermal PMN accumulation by analysis of MPO activity revealed significantly lower levels in IL-8 pretreated mice as compared with BSA pretreated controls $(0.337 \pm 0.056$ vs. $0.960 \pm 0.100$, respectively, $P<0.001$ ) (Fig. 4). These findings supported the hypothesis that the recruitment of neutrophils into the tissue site is an essential step in the pathogenesis of subepidermal blister formation in the experimental model of BP.

Intradermal injection of IL-8 or C5a induces PMN accumulation and confers upon C5-deficient mice susceptibility to the pathogenic effect of anti-mBP180 IgG. As part of a previous study, we had shown that C5-deficient mice were resistant to the pathogenic effects of anti-mBP180 IgG (13). The injected rabbit $\mathrm{IgG}$ and murine $\mathrm{C} 3$ were found deposited at the DEJ of these mice, but there were no signs of neutrophilic infiltration or subepidermal blistering. In an attempt to circumvent this pathogenic block by artificially recruiting neutrophils to the dermis of the C5-deficient mice, the animals were given an intradermal injection of IL-8 or C5a before, or coincident with, the treatment with anti-mBP180 IgG. We showed, by MPO activity assays, that intradermal administration of IL-8 or C5a in these mice resulted in a dose-dependent PMN accumulation at skin sites (data not shown). Significantly, the pathogenic IgG 


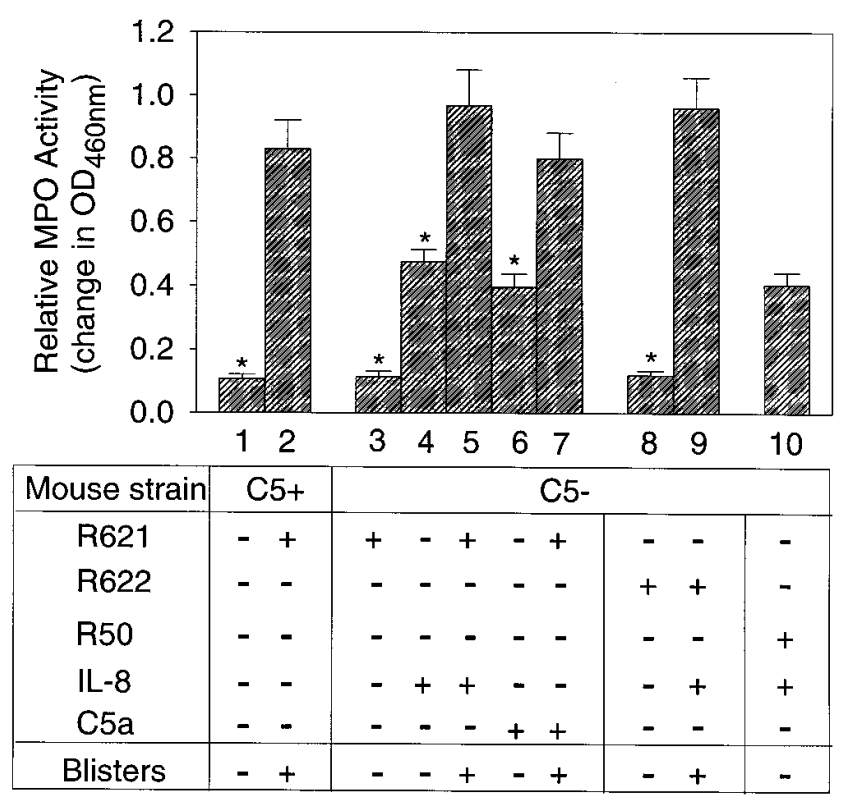

Figure 5. In vivo reconstitution of neutrophils at the tissue site by intradermal injection of IL-8 or C5a restores the pathogenic effect of anti-BP180 IgG in neonatal C5-deficient mice. C5-sufficient (bars 1 and 2) or C5-deficient mice (bars 3-10) were injected intradermally with either pathogenic R621 IgG alone (bars 2 and 3), nonpathogenic R622 IgG alone (bar 8), IL-8 alone (bar 4), or C5a alone (bar 6), or both IgG and chemoattractant (bars 5, 7, 9, and 10). C5-deficient mice coinjected with IL-8 or C5a and pathogenic R621 (bars 5 and 7) as well as nonpathogenic but indirect IF-positive R622 (bar 9), but not the control R50 IgG (bar 10), developed subepidermal blisters. The IgG dose was $2.5 \mathrm{mg} / \mathrm{g}$ body wt. Doses for IL-8 or C5a were 50 $\mathrm{ng}$ /mouse. Tissue MPO activity (mean $\pm \mathrm{SEM}$ ) in skin at the injection site was determined $12 \mathrm{~h}$ after IgG administration. $n=8$ for each group. ${ }^{*} P<0.001$, Student $t$ test for paired samples (bar 1 vs. 2 ; bars 3 and 4 vs. 5 ; bar 6 vs. 7 ; bar 8 vs. 9). See Methods for details.

from the anti-mBP180 serum R621, which is incapable of inducing disease in C5-deficient mice (13), induced extensive blistering in these animals $(n=8)$ after intradermal injection of IL-8 (Table III). Histological examination of the skin of these mice showed typical subepidermal separation and neutrophilic infiltration of the dermis. Interestingly, higher values of MPO activity were obtained from skin extracts of C5-deficient mice coinjected with IL-8 and anti-mBP180 IgG as compared with animals injected with anti-mBP180 IgG alone (Fig. 5). The mean MPO activity level in skin extracts of C5-deficient mice coinjected with anti-mBP180 IgG and IL-8 was $0.968 \pm 0.084 / \mathrm{mg}$ protein (Fig. 5 ). This value is very close to the mean value obtained from $\mathrm{C} 5$-sufficient mice injected with anti-mBP180 IgG alone (0.828 $\pm 0.058 / \mathrm{mg}$ protein), and represents a 9.3-fold increase over the mean value obtained from the mice injected only with anti-mBP180 IgG $(0.104 \pm 0.017$; $P<0.001)$. Very similar results were obtained when C5a was used instead of IL-8 in the passive transfer experiments with C5-deficient and C5-sufficient mice. The control group of C5deficient mice $(n=17)$, which were pretreated with BSA in place of the neutrophil chemoattractant, showed no dermal inflammation or blistering in response to injection with R621 IgG.

We have also reported previously that not all rabbits im- munized with the mBP180 antigen produce pathogenic antibodies (18). For example, R622 IgG, when injected into either $\mathrm{BALB} / \mathrm{c}$ or C5-deficient mice, binds to the DEJ, but does not activate complement nor does it induce an inflammatory skin disease (18, also see Table I). Interestingly, these mice $(n=8)$, when coinjected intradermally with R622 IgG and the neutrophil chemoattractant, IL-8, developed extensive subepidermal blisters with a significant increase of MPO activity at the lesional site $(0.206 \pm 0.025 / \mathrm{mg}$ protein when treated with $\mathrm{R} 622$ $\mathrm{IgG}$ alone vs. $0.878 \pm 0.063 / \mathrm{mg}$ protein when $\mathrm{R} 622 \mathrm{IgG}$ and IL-8 were coinjected, $P<0.001$ ) (Fig. 5). In the final series of experiments, C5-deficient mice $(n=8)$ were coinjected intradermally with R50 IgG and the neutrophil chemoattractant, IL-8. However, R50 IgG, which does not bind to the DEJ (18, also see Table I), did not trigger subepidermal blisters in C5-deficient mice, even when coinjected with IL-8 (Fig. 5).

\section{Discussion}

The aim of this study was to directly assess the role of neutrophils in the pathogenesis of subepidermal blister formation in the experimental model of BP. This animal model involves the injection of neonatal mice with rabbit antibodies that show a restricted specificity for a short stretch of the extracellular domain of the murine BP180 antigen $(12,18)$. Rabbit antibodies recognizing other epitopes of this antigen were found to be harmless in these passive transfer experiments. It was also demonstrated that pathogenic anti-mBP180 antibodies trigger the subepidermal blistering disease only when the complement cascade was intact and active (13). Thus, the pathogenicity of rabbit anti-mBP180 antibodies is dependent on both the fine specificity and the complement fixing properties of these IgG molecules. In this report, we define a major role for the complement system and the relevance of neutrophils as effector cells in anti-mBP180-mediated lesion formation.

To test the role of neutrophils, we first documented the sequential in vivo events of immune complex formation, complement activation, neutrophil influx, and dermal-epidermal separation by a time course study. The clinical and immunohistological findings as well as the levels of MPO activity in extracts of lesional and nonlesional skin of these mice were established at each time point. The results of these studies demonstrated a direct correlation between neutrophilic infiltration (as quantified by MPO activity levels at the lesional site) and the degree of subepidermal blistering after local (intradermal) or systemic (intraperitoneal) injection of antimBP180 IgG. Subepidermal blistering mediated by antimBP180 antibodies was triggered more rapidly (as determined by both visual and microscopic examinations) when the $\mathrm{IgG}$ fraction was administered by intradermal versus intraperitoneal injection, presumably due to the speed of access to its target antigen (i.e., the BP180 protein) and the local IgG concentration effect.

To determine whether neutrophils play an essential role in anti-mBP180 induced subepidermal blistering, neonatal BALB/c mice were made neutropenic by two depletion methods, one by treatment of the animals with a cytotoxic antineutrophil antiserum, and another by intraperitoneal injections of IL-8, which sequesters neutrophils in the peritoneal cavity. Both of these methods were shown to be effective in inducing neutropenia in the experimental animals (23-27). In both experimental groups, the mice became resistant to the pathogenic effects 
of the anti-mBP180 $\mathrm{IgG}$, despite the fact that rabbit anti$\mathrm{mBP} 180 \mathrm{IgG}$ reached high titers in the circulation of these animals and was deposited at the cutaneous BMZ along with murine complement. As expected, neutrophilic infiltration of the dermis of these animals was minimal or absent, as determined by $\mathrm{H} / \mathrm{E}$ and MPO analyses. These findings provided strong support for the hypothesis that neutrophilic infiltration to the target tissue is an obligatory step in the formation of subepidermal blisters induced by pathogenic anti-mBP180 antibodies.

To further investigate this hypothesis, a series of passive transfer experiments was performed on C5-deficient mice. In agreement with the findings from a previous study by our research group (13), intradermal injection of anti-BP180 antibodies into these mice resulted in the deposition of rabbit IgG and murine $\mathrm{C} 3$ at the DEJ, but did not induce neutrophilic infiltration of the dermis nor any signs of blistering disease. Significantly, when a potent neutrophil chemoattractant, such as IL- 8 or C5a, was coadministered with the anti-mBP180 antibodies, the C5-deficient mice showed both clinical and histological evidence of neutrophilic infiltration of the dermis and subepidermal blistering. The formation of these cutaneous lesions was dependent upon the presence of both the anti$\mathrm{mBP} 180 \mathrm{IgG}$ and the neutrophil chemoattractant, since intradermal injection of the chemoattractant (without anti-mBP180 antibodies) induced intensive neutrophilic infiltration of the dermis without subepidermal blister formation in both C5deficient and C5-sufficient mice. It has been shown previously that intradermal injections of these chemoattractants induce a dose-dependent accumulation of neutrophils in the injected dermal sites of the skin of rats $(28)$, rabbits $(29,30)$, humans $(31,32)$, and mice $(33,34)$.

Our results with the $\mathrm{C} 5$-deficient mice provide further support for the conclusion that neutrophil recruitment to the DEJ is an essential step in anti-mBP180-induced subepidermal blistering. Moreover, the findings from these neutrophil reconstitution experiments suggest that the main role of C5a in subepidermal blister formation is to recruit neutrophils to the target tissue site. This function of C5a can be carried out via a direct effect on neutrophils and/or by upregulating the expression of other neutrophil chemoattractants, such as IL-8. In fact, this dual function of C5a has been documented previously $(35,36)$. Since administration of C5a or IL-8 to C5-deficient mice reverses the animals' resistance to the pathogenic effects of anti$\mathrm{mBP} 180 \mathrm{IgG}$, this suggests that the membrane attack complex (C5-9) does not play a key role in the initial stages of tissue damage triggered by anti-mBP180 IgG.

In a final series of experiments, we demonstrated that antimBP180 antibodies present in serum R622, which are nonpathogenic by passive transfer experiments, become pathogenic, if coinjected intradermally with $\mathrm{C} 5 \mathrm{a}$ or IL-8. It was reported previously that anti-mBP180 antibodies of both R621 and R622 sera bound to the cutaneous BMZ of BALB/c mice, but only R621 was able to fix murine $\mathrm{C} 3$ and promote neutrophilic infiltration of the dermis and subepidermal blistering (18). These observations strongly suggest that the functional difference between the anti-mBP180 antibodies of R621 and R622 sera, in terms of triggering subepidermal blistering, lies in their capacity to activate complement in vivo. It is also feasible that the differences exhibited by these two anti-mBP180 preparations may be due to differences in ability to stimulate local cells (presumably basal keratinocytes or mast cells) to re- lease proinflammatory mediators which subsequently lead to complement activation and neutrophilic infiltration. Support for this scenario was provided by our recent molecular mapping study of the pathogenically relevant BP180 epitope (18). We showed that R621 and R622 IgG reacted with distinct sites on the BP180 antigen. In these studies, we further demonstrated that R50 IgG, which binds to denatured BP180 antigen as determined by immunoblotting, but which does not bind to native BP180 as determined by direct and indirect IF, failed to trigger subepidermal blistering in mice when coinjected with IL-8.

Despite the striking similarities in the immunopathological features of the experimental model of BP and the human disease, there is one conspicuous difference. The inflammatory infiltrate of early lesions in human BP shows large numbers of eosinophils (1), while in the mouse model, neutrophils are the predominant inflammatory cell type in the perilesional sites (12). The time course study uncovered no signs of eosinophil recruitment into the injection site of the mice up to $24 \mathrm{~h}$ after passive transfer of pathogenic anti-mBP180 IgG, while extensive subepidermal blistering was seen by $12 \mathrm{~h}$ after injection. Therefore, it is quite clear that in experimental BP, eosinophils do not play an important role in the initial stages of subepidermal blistering. In fact, neutrophil-predominant forms of BP have been described in which eosinophils are absent in the inflammatory cell infiltrate (37). These patients suggest that eosinophils are not a requirement for blister development. It is worth noting that most patient biopsies are obtained $>24 \mathrm{~h}$ after the initial onset of disease activity. It is possible that if the mouse model were maintained long term that eosinophils might be recruited in a more chronic phase. While at this point we cannot rule out the possibility that there are critical differences in the pathogenic mechanisms of human BP and the BP mouse model, it remains quite possible, given the available information, that eosinophils are not directly involved, or play only a secondary role, in the initiation of human BP. These cells may well be important in later stages of the disease, e.g., wound healing after tissue damage.

In summary, this study provides the first direct evidence that neutrophils are indeed important effector cells in the pathogenesis of blister formation in the experimental model of $\mathrm{BP}$. This conclusion is further reinforced by the finding that subepidermal blistering in the experimental BP model is mediated by molecular interaction between Fc of anti-BP180 IgG and Fc receptors of neutrophils and is dependent upon neutrophil elastase activity (Liu, Z., S.A. Twining, X. Zhou, G.J. Giudice, J.A. Fairley, and L.A. Diaz, manuscript submitted for publication; Liu, Z., X. Zhou, G.J. Giudice, J.A. Fairley, and L.A. Diaz, manuscript in preparation). In this study we also showed that C5a has an essential function in this pathogenic mechanism which involves recruiting neutrophils to the target tissue site. In addition to shedding new light on the pathogenesis of $\mathrm{BP}$, these findings suggest new directions for disease intervention.

\section{Acknowledgments}

This work was supported in part by U.S. Public Health Service grants R29 AI40768 (Z. Liu), R01 AR-40410 (G.J. Giudice), and R01 AR32599 and R37-AR32081 (L.A. Diaz) from the National Institutes of Health and by a VA Merit Review Grant (L.A. Diaz). Z. Liu was the recipient of a Dermatology Foundation Career Development Award 
and a Dermatology Foundation Research Grant sponsored by the Burroughs Wellcome Fund.

\section{References}

1. Korman, N.J. 1993. Bullous pemphigoid. Dermatologic Clinics. 11:483-498.

2. Lever, W.F. 1953. Pemphigus. Medicine (Baltimore). 32:1-123.

3. Emmerson, R.W., and E. Wilson-Jone. 1968. Eosinophilic spongiosis in pemphigus. A report of an unusual histological change in pemphigus. Arch. Dermatol. 97:252-257.

4. Nishioka, K., K. Hashimoto, I. Katayama, C. Sarashi, T. Kubo, and S. Sano. 1984. Eosinophilic spongiosis in bullous pemphigoid. Arch. Dermatol. 120:1166-1168.

5. Natio, K., S. Morioka, S. Ikeda, and H. Ogawa. 1984. Experimental bullous pemphigoid in guinea pigs: the role of pemphigoid antibodies, complement, and migrating cells. J. Invest. Dermatol. 82:227-230.

6. Nestor, M.S., A.J. Cochran, and A.R. Ahmed. 1987. Mononuclear cell infiltrates in bullous disease. J. Invest. Dermatol. 88:172-175.

7. Dubertret, L., B. Bertaux, M. Fosse, and R. Touraine. 1980. Cellular events leading to blister formation in bullous pemphigoid. Br. J. Dermatol. 104: 615-624.

8. Iwatsuki, K., H. Tagami, and M. Yamada. 1983. Induction of leukocyte adherence at the basement membrane zone with subsequent activation of their metabolic pathway by pemphigoid antibodies and complement. Acta DermatoVenereol. 63:495-500.

9. Wintroub, B.U., M.C. Mihm, Jr., E.J. Goetzl, N.A. Soter, and K.F. Austen. 1978. Morphologic and functional evidence for release of mast-cell products in bullous pemphigoid. N. Engl. J. Med. 298:417-421.

10. Dvorak, A.M., M.C. Mihm, Jr., J.E. Osage, T.H. Kwan, K.F. Austen, and B.U. Wintroub. 1982. Bullous pemphigoid, an ultrastructural study of the inflammatory response: eosinophil, basophil and mast granule changes in multiple biopsies from one patient. J. Invest. Dermatol. 78:91-101.

11. Shornick, J.K., J.L. Bangert, R.G. Freeman, and J.N. Gilliam. 1983. Herpes gestationis: clinical and histological features of twenty-eight cases. $J$. Am. Acad. Dermatol. 8:214-224.

12. Liu, Z., L.A. Diaz, J.L. Troy, A.F. Taylor, D.E. Emery, J.A. Fairley, and G.J. Giudice. 1993. A passive transfer model of the organ-specific autoimmune disease, bullous pemphigoid, using antibodies generated against the hemidesmosomal antigen, BP180. J. Clin. Invest. 92:2480-2488.

13. Liu, Z., G.J. Giudice, S.J. Swartz, J.A. Fairley, G.O. Till, J.L. Troy, and L.A. Diaz. 1995. The role of complement in experimental bullous pemphigoid. J. Clin. Invest. 95:1539-1544.

14. Gammon, W.R., D.M. Lewis, J.R. Carlo, W.M. Sams, Jr., and C.E. Wheeler, Jr. 1980. Pemphigoid antibody-mediated attachment of peripheral blood leukocytes at dermal-epidermal junction of human skin. J. Invest. Dermatol. 75:334-339.

15. Gammon, W.R., C.C. Merritt, D.M. Lewis, W.M. Sams, Jr., J.R. Carlo, and C.E. Wheeler, Jr. 1982. An in vitro model of immune complex-mediated basement membrane zone separation caused by pemphigoid antibodies, leukocytes, and complement. J. Invest. Dermatol. 78:285-290.

16. Li, K., K. Tamai, E.M.L. Tan, and J. Uitto. 1993. Cloning of type XVII collagen. Complementary and genomic DNA sequences of mouse 180-kilodalton bullous pemphigoid antigen (BPAG2) predict an interrupted collagenous domain, a transmembrane segment, and unusual features in the $5^{\prime}$-end of the gene and the $3^{\prime}$-untranslated region of the mRNA. J. Biol. Chem. 268:88258834 .

17. Liu, Z., L.A. Diaz, A.L. Haas, and G.J. Giudice. 1992. cDNA cloning of a novel human ubiquitin carrier protein. An antigenic domain specifically recognized by endemic pemphigus foliaceus autoantibodies is encoded in a secondary reading frame of this human epidermal transcript. J. Biol. Chem. 267: 15829-15835.

18. Liu, Z., L.A. Diaz, S.J. Swartz, J.L. Troy, J.A. Fairley, and G.J. Giudice.
1995. Molecular mapping of a pathogenically relevant BP180 epitope associated with experimentally induced murine bullous pemphigoid. J. Immunol. 155: 5449-5454.

19. Anhalt, G.J., R.S. Labib, J.J. Voorhees, T.F. Beals, and L.A. Diaz. 1982. Induction of pemphigus in mice by passive transfer of $\mathrm{IgG}$ from patients with the disease. N. Engl. J. Med. 306:1189-1196.

20. Roscoe, J.T., L.A. Diaz, S.A.P. Sampaio, R.M. Castro, R.S. Labib, H. Patel, and G.J. Anhalt. 1985. Brazilian pemphigus foliaceus autoantibodies are pathogenic to BALB/c mice by passive transfer. J. Invest. Dermatol. 85:538-541.

21. Bradley, P.P., D.A. Priebat, R.D. Christensen, and G. Rothstein. 1982. Measurement of cutaneous inflammation: estimation of neutrophil content with an enzyme marker. J. Invest. Dermatol. 78:206-209.

22. Mulligan, M.S., M.L. Jones, M.A. Bolanowski, M.P. Baganoff, C.L. Deppeler, D.M. Meyers, U.S. Ryan, and P.A. Ward. 1993. Inhibition of lung inflammatory reactions in rats by an anti-human IL-8 antibody. J. Immunol. 150: $5585-5595$.

23. Hechtman, D.H., M.I. Cybulsky, H.J. Fuchs, J.B. Baker, and M.A. Gimbrone, Jr. 1991. Intravascular interleukin-8: an inhibitor of polymorphonuclear leukocyte accumulation at sites of acute inflammation. J. Immunol. 147:883-892.

24. Ley, K., J.B. Baker, M.I. Cybulsky, M.A. Gimbrone, Jr., and F.W. Luscinskas. 1993. Intravenous interleukin-8 inhibits granulocyte emigration from rabbit mesenteric venules without altering L-selectin expression or leukocyte rolling. J. Immunol. 151:6347-6357.

25. Till, G.O., K.J. Johnson, R. Kunkel, and P.A. Ward. 1982. Intravascular activation of complement and acute lung injury. J. Clin. Invest. 69:1126-1135.

26. Sekiya, S., S. Gotoh, T. Yamashita, T. Watanabe, S. Saitoh, and F. Sendo. 1989. Selective depletion of rat neutrophils by in vivo administration of a monoclonal antibody. J. Leukocyte Biol. 46:96-102.

27. Rogers, H.W., and E.R. Unanue. 1993. Neutrophils are involved in acute, nonspecific resistance to Listeria monocytogenes in mice. Infect. Immun. 61:5090-5096.

28. Ribeiro, R.A., C.A. Flores, F.Q. Cunha, and S.H. Ferreira. 1991. IL-8 causes in vivo neutrophil migration by a cell-dependent mechanism. Immunology. 73:472-477.

29. Colditz, I., R. Zwahlen, B. Dewald, and M. Baggiolini. 1989. In vivo inflammatory activity of neutrophil-activating factor, a novel chemotactic peptide derived from human monocytes. Am. J. Pathol. 134:755-760.

30. Foster, S.J., D.M. Aked, J.-M. Schröder, and E. Christophers. 1990. Acute inflammatory effects of a monocyte-derived neutrophil-activating peptide in rabbit skin. Immunology. 67:181-183.

31. Swensson, O., C. Schubert, E. Christophers, and J.-M. Schröder. 1991. Inflammatory properties of neutrophil-activating protein-1/interleukin 8 (NAP1/IL-8) in human skin: a light- and electron microscopic study. J. Invest. Dermatol. 96:682-689.

32. Leonard, E.J., T. Yoshimura, S. Tanaka, and M. Raffeld. 1991. Neutrophil recruitment by intradermally injected neutrophil attractant/activating protein-1. J. Invest. Dermatol. 96:690-694.

33. Rot, A. 1991. Chemotactic potency of recombinant human neutrophil attractant/activation protein-1 (interleukin-8) for polymorphonuclear leukocytes of different species. Cytokine. 3:21-27.

34. Hiltz, E., A. Catania, and J.M. Lipton. 1992. $\alpha$-MSH peptides inhibit acute inflammation induced in mice by rIL-1 $\beta$, rIL- 6, rTNF- $\alpha$ and endogenous pyrogen but not that caused by LTB4, PAF and rIL-8. Cytokine. 4:320-328.

35. Fernandez, H.N., P.H. Henson, A. Otani, and T.E. Hugli. 1979. Chemotactic response to human $\mathrm{C} 3 \mathrm{a}$ and $\mathrm{C} 5 \mathrm{a}$ anaphylatoxins. I. Evaluation of $\mathrm{C} 3 \mathrm{a}$ and C5a leukotaxis in vitro and under stimulated in vivo conditions. J. Immunol. 120:109-115.

36. Ember, J.A., S.D. Sanderson, T.E. Hugli, and E.L. Morgan. 1994. Induction of interleukin-8 synthesis from monocytes by human C5a anaphylatoxin. Am. J. Pathol. 144:393-403.

37. Ackerman, A.B., N. Chongchitnant, J. Sanchez, Y. Guo, B. Rennin, M. Reichel, and M.B. Randal. 1997. Histologic Diagnosis of Inflammatory Skin Diseases. 2nd Edition. Williams and Wilkins, Baltimore. 238-239. 